\title{
On the Trapped Surface Characterization of the Black Hole Region in Kerr Spacetime
}

\author{
Mohammed Kumah ${ }^{1} \&$ Francis T. Oduro ${ }^{2}$ \\ ${ }^{1}$ Department of Mathematics, Kwame Nkrumah University of Science and Technology, Kumasi, Ghana \\ ${ }^{2}$ Department of Mathematics, Kwame Nkrumah University of Science and Technology, Kumasi, Ghana \\ Correspondence: Mohammed Kumah, PMB, Department of Mathematics, Kwame Nkrumah University of Science and \\ Technology, Kumasi, Ghana. E-mail: kumtheta@hotmail.com
}

Received: December 22, 2017 Accepted: January 8, 2018 Online Published: May 28, 2018

doi:10.5539/jmr.v10n4p24 URL: https://doi.org/10.5539/jmr.v10n4p24

\begin{abstract}
Black holes are classically characterized by event horizon which is the boundary of the region from which particles or photons can escape to infinity in the future direction. Unfortunately this characterization is a global concept as the knowledge of the whole spacetime is needed in order to locate a black hole region and the event horizon. It is therefore important to recognize black holes locally; this has motivated the need to use local approach to characterize black holes. Specifically, we apply covariant divergence and Gausss divergence theorems to compute the divergences and the fluxes of appropriate null vectors in the Kerr spacetime to actually determine the existence of trapped and marginally trapped surfaces in its black hole region.
\end{abstract}

Keywords: Trapped surfaces, black holes, event horizons

\section{Introduction}

One of the most striking results of General Relativity is its prediction of black holes which are spacetime regions from which no signal can be seen by an observer far from the matter sources (Frolov and Zelnikov, 2011). These Black holes are formed through the gravitational collapse of sufficiently massive objects, such as massive stars as demonstrated by the work of Chandrasekhar in 1983. General relativity shows that black holes are remarkably simple objects which are characterized by just a few numbers. As stated by Chandrasekhar the black holes of nature are the most perfect macroscopic objects there are in the universe: the only elements in their construction are our concepts of space and time (Hartle, 2003). The existence of black holes was first discussed by Michell and Laplace within the framework of the Newtonian theory at the end of the 18th century (Frolov and Zelnikov, 2011). It was then viewed as a star with strong gravitational field so that the Newtonian escape velocity $\sqrt{2 G M / R}$ (with $M$ and $R$ being the mass and radius of the star respectively) is larger than the speed of light. In fact, the inequality $R \leq 2 G M / C^{2}$ for escape velocity holds in general relativity (Penrose, 2004; Krishnan, 2013).

Kerr discovered a solution of the Einstein equation, which describes the gravitational field of a stationary rotating black hole in 1963 (Jakobsson, 2017). This solution has a gravitational radius which prescribes the position of the event horizon (Frolov and Zelnikov, 2001). Carter explained its global properties in 1966 (Booth, 2005). The Kerr-Newman solution which represents charged spinning black holes was discovered in1965. John Wheeler introduced the term black hole in 1967 (Frolov and Zelnikov, 2011; Krishnan, 2012). There were seminal developments at that very helping to understand the general properties of black holes. These included the study of the global properties of black hole spacetimes, the definition of event horizon, the singularity theorems of Penrose and Hawking as well as the introduction of the concept of trapped surfaces by Penrose (Krishnan, 2012). The black hole uniqueness theorems which showed that the Kerr-Newman solutions are the unique globally stationary black hole solutions in the Einstein-Maxwell theory in four dimensions was established in the 1980s following the work of Israel, Carter and Robinson (Krishnan, 2013). In the 1980s, Robinson and Carter (Krishnan, 2013) established the uniqueness theorems of the Kerr Newman solutions for the description of the black holes of nature. This theorem states that; stationary axisymmetric solutions of Einstein's equation for the vacuum, which have a smooth convex event horizon, are asymptotically flat and are non-singular outside of the horizon, are uniquely specified by the two parameters, the mass and the angular momentum and these two parameters only (Chandrasekhar, 1983).

These theorems assert that, given a matter model, a static or a stationary black hole spacetime belongs necessarily to a specific class of spacetimes (in the vacuum case, they are Schwarzschild in the static regime and Kerr for the stationary case) which are characterized by a few parameters that describe the fundamental properties of the black hole. Thus, 
the Kerr solution represents the unique solution which the general theory of relativity provides for the description of all black holes that can occur in the astronomical universe by the gravitational collapse of stellar masses; and it is the only instance of a physical theory providing an exact description of a macroscopic object (Chandrasekhar, 1983). The study of black holes has for many years depended on event horizons as the boundary of the region of the black hole from where one can send signals to infinity (Senovilla, 2011). However, the study of black holes based on the concept of classical event horizon has the following drawbacks: to locate a black hole region and event horizon requires the knowledge of the entire spacetime and the definition has no direct relation with the notion of strong gravitational field as shown by (Ashtekar and Krishnan, 2004) and (Krishnan, 2013). For example in the Vaidya spacetime, event horizon can form in a flat region. Another global feature of event horizons is their teleological nature (Gourgoulhon and Jaramillo, 2008). The classical black hole boundary, i.e. the event horizon, responds in advance to what will happen in the future. Booth (2005) showed this using the explicit example of a black hole formed by the collapse of two successive matter shells: after the first shell has collapsed to form the event horizon, the latter remains stationary for a while and then starts to grow before the second collapsing shell reaches it (Gourgoulhon and Jaramillo, 2008). If black holes are considered as "ordinary" physical objects, for instance in quantum gravity or numerical relativity, the above mentioned global behaviour of the event horizon would be problematic (Gourgoulhon and Jaramillo, 2008).

The global nature of the event horizon and these physical problems associated with the event horizon have motivated the need to use a local approach as a complementary means of characterizing black holes. Through the Hawking and Penrose's singularity theorems and weak cosmic censorship, the existence of a black hole region is indicated. In fact, in strongly predictable spacetimes satisfying proper energy conditions, trapped surfaces are guaranteed to lie inside the black hole region. Moreover; their location does not involve a whole future spacetime development (Jaramillo, 2011). The purpose of this paper is to explicitly demonstrate the existence of trapped surfaces and marginally trapped surfaces by computing the expansions of null vectors (Krishnan, 2003) in the Kerr black hole region.

The plan of the paper is as follows: section 2 discusses the covariant divergence which is the main tool for the computation of the divergences of both ingoing and outgoing null vectors. We also discuss Gauss divergence theorem for the computation of the flux of a vector field. This section also contains discussions of the local characterizations of black holes. A general discussion of the Kerr black hole in Boyer-Lindquist coordinate systems are presented in the same section. In section 3, the existence of trapped and marginally trapped surfaces in the Kerr black hole is discussed after computing their covariant divergences. In this same section, we apply Gauss' divergence theorem to compute the fluxes of vector fields to support the claim that trapped and marginally trapped surfaces exist in the Kerr black hole. This is the main result of this paper. Section 4 then gives the conclusion of the result.

\section{Local Characterizations of Black Holes}

\subsection{Trapped Surface}

A two-dimensional surface $\mathrm{S}$ in a four dimensional spacetime has two null directions normal to the surface at each point. Trapped surfaces are characterized via the covariant divergences of such vectors which are orthogonal to the surface. We can thus distinguish two future directed null vectors emerging from the surface $S$. If we denote the in-going and out-going null normals to the surface $S$ by $l^{\alpha}$ and $n^{\alpha}$ respectively, then $\theta_{l}$ and $\theta_{n}$ are their respective divergences.

The surface $S$ is said to be trapped if both divergences are negative: $\theta_{l}<0$ and $\theta_{n}<0$ or according to Penrose, a trapped surface $\mathrm{S}$ is a compact, space-like 2-dimensional sub-manifold of space-time on which $\theta_{l} \theta_{n}>0$ (Ashtekar and Krishnan, 2004).In flat space, the out-going light rays diverge and the ingoing ones converge, i.e. $\theta_{l}>0$ and $\theta_{n}<0$ so trapped surface cannot exist there. The notion of trapped surfaces, due to Penrose (1965), entails that, in a sufficiently strong gravitational field, as in gravitational collapse, even outgoing light rays converge.

In stationary black holes,such as the Schwarzschild black hole, the event horizon and the Killing horizon are identical and characterize the boundary of the region which contains trapped surfaces (Booth, Kunduri, and OGrady, 2017). In dynamical black holes, however, trapped surfaces are generally separated from the event horizon and located inside the apparent horizon (Ben-Dov, 2007). This means it is always possible to locate trapped surfaces inside the black hole region. These trapped surfaces are central to the singularity theorems and their presence indicate the development of singularity and therefore the formation of black holes (Senovilla, 2011).Trapped surfaces are also important especially because they are independent of the coordinates and of the existence of symmetries such as spherical or axial such as present in Kerr's solution. More importantly, trapped surface defined by the inequalities $\theta_{l}<0$ and $\theta_{n}<0$, remain stable even under small perturbation (Senovilla and Garfinkle, 2015). It is therefore of interest to investigate the relation between the event horizon and various notions of black holes boundaries based on a special reference to trapped surfaces. 


\subsection{Marginally Trapped Surface}

The surface $S$ is said to be a marginally trapped surface (MTS) if $\theta_{l}=0$ and $\theta_{n}<0$. The singularity theorems (Hawking and Penrose, 1970, Senovilla, 1998a) have shown that the presence of such surfaces is the signature of a spacetime containing a black hole. Hayward, (1994) defines a marginally trapped surface as a spatial 2-surface S on which one null expansion vanishes

\subsection{The Trapped Region and the Trapping Boundary}

An outer trapped surface is defined by Hawking as a compact spacelike 2-dimensional submanifold in $\left(\mathrm{M}, g_{a b}\right)$ such that the expansion of the outgoing null geodesics orthogonal to the surface is non-positive (Ashtekar and Krishnan, 2004). With regard to this definition, it does not matter whether the ingoing null geodesics are converging or not. However, for convenience the case $\theta=0$ is included.

Trapped region: Hawking defines the trapped region denoted $T(M)$ in a surface $M$ as the set of all points in $M$, through which there passes an outer-trapped surface, lying entirely in $M$. The spacetime region $T$ containing trapped surface is called the trapped region (Ashtekar and Krishnan, 2004). Schnetter and Krishnan (2006) define a trapped region as the region where trapped surfaces exist, as can be found in the full spacetime or on a Cauchy surface. (Hayward, 1994) defines a trapped region as a subset of space-time through each point of which there passes a trapped surface. Trapping boundary is defined as a connected component of the boundary of an inextendible trapped region Hayward (1994). A trapping horizon is defined as a hypersurface of $\mathrm{M}$ which is foliated by spacelike 2-surfaces $\mathrm{S}$ such that the expansion scalar $\theta_{l}$ of one of the null geodesics orthogonal to $\mathrm{S}$ vanishes. A trapping horizon can be spacelike or null (Hayward, 1994).

\subsection{Covariant Divergence of a Vector Field}

The covariant divergence of a vector field is given by

$$
\operatorname{div} \bar{V}=\partial_{i} V^{i}+\Gamma_{i k}^{i} V^{k}
$$

which is a scalar in all frames and reduces to the familiar form in a Cartesian system where

$$
\Gamma_{i j}^{i}=\frac{1}{2} g^{i k}\left[\frac{\partial g_{j k}}{\partial x^{i}}+\frac{\partial g_{k i}}{\partial x^{j}}-\frac{\partial g_{i j}}{\partial x^{k}}\right]
$$

Thus the covariant divergence can be written compactly as

$$
\operatorname{div} \bar{V}=\nabla_{i} V^{i}=\frac{\partial V^{i}}{\partial x^{i}}+\frac{1}{\sqrt{g}} \frac{\partial \sqrt{g}}{\partial x^{j}} V^{i}=\frac{1}{\sqrt{g}}\left[\sqrt{g} \frac{\partial V^{i}}{\partial x^{i}}+\frac{\partial \sqrt{g}}{\partial x^{j}} V^{i}\right]=\frac{1}{\sqrt{g}} \frac{\partial\left(\sqrt{g} V^{i}\right)}{\partial x^{i}}
$$

In this compact form, one only needs to calculate $\mathrm{g}$ and the derivatives specified above, but not the Christoffel symbols directly to evaluate the covariant divergence of a vector field. (Kumah and Oduro, 2018)

\subsection{Existence of Trapped and Marginally Trapped Surfaces Using Gauss's Divergence Theorem}

Theorem 2.1; The Gauss' divergence theorem: The integral of the divergence of a vector field over a region $V$ equals the flux of the field through the surface $S$ bounding $V$ provided the field is suitably smooth inside $V$ and $S$ (Borisenko and Tarapov 1979). If we consider region $V$, in which a vector field $\bar{g}$ is continuous and differentiable, the divergence of this vector field is given by

$$
\int_{V} \nabla \cdot \bar{A} d V=\oint_{S} \bar{A} \cdot \bar{n} d S
$$

Where the surface $S$ is a closed surface that completely surrounds a very small volume region $V$ at point $r$.

Remark 2.2: The divergence basically indicates the amount of vector field $\bar{g}$ that is converging to or diverging from a given point. Hence if there is a massive source enclosed by the surface, its gravitational field has an attractive or converging effect. Close enough to a massive source; the outgoing null vectors converge and the flux becomes negative i.e. $\nabla \cdot \bar{l}<0$. We recall that a surface $S$ is said to be trapped if and only if both fluxes are negative: i.e. $\nabla \cdot \bar{l}=\theta_{l}<0$ and $\nabla \cdot \bar{n}=\theta_{n}<0$. The surface where the flux of outgoing null vector becomes zero and the flux of ingoing null vector is less than zero is then said to be marginally trapped (MTS) i.e. $\nabla \cdot \bar{l}=\theta_{l}=0$ and $\nabla \cdot \bar{n}=\theta_{n}<0$.

\subsection{The Kerr Spacetime}

The Kerr metric represents the unique solution which the general theory of relativity provides for the description of all black holes that can occur in the astronomical universe by the gravitational collapse of all stellar masses and it is 
the only instance of a physical theory providing an exact description of a macroscopic objects (Chandrasekhar, 1983). Quantitatively the character of Kerr spacetime depends on its mass and the rate of its rotation, especially when the rotation is fairly slow. The Kerr spacetime reduces to the Schwarzschild spacetime if the rotation stops entirely. Inside the first horizon of this Kerr black hole can be found a second horizon. Behind this horizon, there exists the singularity of the spacetime as a ring which is a circle of infinite gravitational forces. This singularity is not as serious as the one in the Schwarzschild spacetime. It can be avoided when entering a new region of spacetime by passing through either of the two throats bounded by the ring. It is easy to escape the ring singularity from the new region because the black hole gravitational effect is reversed so it rather repels than attracts (ONeill, 1995).

\subsection{The Kerr Spacetime in Boyer-Lindquist Coordinates}

The Kerr metric looks very simple in Boyer-Lindquist coordinates $(t, r, \theta, \phi)$ because of the minimization of the number of off-diagonal components. These coordinates help us to analyze the asymptotic behaviour and understand the main difference between an "event horizon" and an "ergosphere" (ONeil, 1995). In these coordinates the metric has only one off-diagonal component and takes the form.

$$
\begin{aligned}
d s^{2}= & -\left(1-\frac{2 M r}{\rho^{2}}\right) d t^{2}-\frac{4 M a r \sin ^{2} \theta}{\rho^{2}} d t d \phi+\frac{\Sigma}{\rho^{2}} \sin ^{2} \theta \phi^{2}+\frac{\rho^{2}}{\Delta} d r^{2}+ \\
& \rho^{2} d \theta^{2} \\
= & -\frac{\rho^{2} \Delta}{\Sigma} d t^{2}+\frac{\Sigma}{\rho^{2}} \sin ^{2} \theta(d \phi-\omega d t)^{2}+\frac{\rho^{2}}{\triangle} d r^{2}+\rho^{2} d \theta^{2}
\end{aligned}
$$

where $a=J / M, \rho^{2}=r^{2}+a^{2} \cos ^{2} \theta, \Delta=r^{2}-2 M r+a^{2}, \Sigma=\left(r^{2}+a^{2}\right)^{2}-a^{2} \Delta \sin ^{2} \theta, \omega=-\frac{g_{t \phi}}{g_{\phi \phi}}=\frac{2 M a r}{\Sigma}$

The spin parameter $a$, is the Kerr parameter which has units of length in geometrized units just like the mass. The parameter $J$ is interpreted as spacetime total angular momentum and the parameter $\mathrm{M}$ as the spacetime total mass (Gourgoulhon,2017). The Kerr metric has the following properties. That it is not static implies it is not invariant under time reversal. It is stationary and does not depend explicitly on time $t$. It is axisymmetric and does not depend explicitly on $\phi$. This metric form is clearly invariant under simultaneous inversion of $t$ and $\phi$, i.e. under the transformation $t \rightarrow-t, \phi \rightarrow-\phi$ although it is not invariant under inversion of $t$ alone (except when $a=0$ ). It is a vacuum solution of the Einstein equations, valid in the absence of matter. Since the metric is stationary and axially symmetric, it therefore admits the Killing vectors $t^{\alpha}=\frac{\partial x^{\alpha}}{\partial t}$ and $\phi^{\alpha}=\frac{\partial x^{\alpha}}{\partial \phi}$ (Carroll, 2014). If the black hole is not rotating i.e. $a=J / M=0$, the Kerr line metric reduces to the Schwarzschild metric (Ashtekar and Galloway, 2005).

\subsection{Singularities of the Kerr Spacetime}

The Kerr metric as shown by (5) diverge at two locations given by

(i) $\rho^{2}=0$ corresponding to a curvature singularity. The Kretschmann scalar (Carroll, 2014) of this metric is $R_{\mu \nu \rho \sigma} R^{\mu \nu \rho \sigma}=$ $\frac{48 M^{2}\left(r^{2}-a^{2} \cos ^{2} \theta\right)\left(\rho^{4}-16 a^{2} r^{2} \cos ^{2} \theta\right)}{\rho^{12}}$ which diverge for $r \neq 0$ (i.e $\rho^{2}=0$ ). This curvature singularity cannot be reduced by any coordinate system.

(ii) $\Delta=0$ : corresponding to a coordinate singularity, i.e. a pathology of Boyer-Lindquist coordinates, which can be reduced by using other coordinate systems such as Eddington-Finkelstein coordinates (Gourgoulhon, 2017).

\subsection{Event Horizon of the Kerr Spacetime}

The system of Boyer-Lindquist coordinates $(t, r, \theta, \phi)$ breaks down when $\Delta=0$ i.e. on a possible horizon. This means the event horizons of the Kerr black hole occur at $\Delta=r^{2}-2 M r+a^{2}=0$ and $r_{ \pm}=M \pm \sqrt{M^{2}-a^{2}}$ where $r_{+}$corresponds to the horizon that makes the Kerr solution a black hole. The event horizons of the Kerr spacetime are null 3-dimensional surfaces and their spatial slices have the geometry of a 2-dimensional distorted sphere. The Kerr solution with $a^{2}>M^{2}$ contains naked singularities (In this case the singularity would be observable) and so are not regular predictable spaces (Hawking and Ellis 1973). This type of Kerr solution does not have an event horizon and so does not describe a black hole. It has been conjectured that the solution outside an uncharged collapsed object will settle down to a Kerr solution with $a^{2} \leq M^{2}$. This conjecture is supported by (Hawking and Ellis, 1973). 


\section{Trapped Surface and Marginally Trapped Surface in the Kerr Spacetime}

\subsection{Trapped Surface in the Kerr Black Hole Identified Using the Covariant Divergence of a Vector Field}

In (Krishnan, 2013), a suitable choice of the ingoing and outgoing future directed null vectors for non-extremal Kerr black hole are

$$
\begin{aligned}
l^{\mu} \nabla \mu & =\frac{\partial}{\partial v}+\frac{\Delta}{2\left(r^{2}+a^{2}\right)} \frac{\partial}{\partial r}+\frac{a}{r^{2}+a^{2}} \frac{\partial}{\partial \phi} \\
l^{\mu} \nabla \mu & =-\left(\frac{r^{2}+a^{2}}{\rho^{2}}\right) \frac{\partial}{\partial r}
\end{aligned}
$$

The covariant versions are

$$
\begin{aligned}
& l_{\mu}=-\frac{\Delta}{2\left(r^{2}+a^{2}\right)}\left(\frac{\partial}{\partial v}\right)^{\mu}+\frac{\rho^{2}}{r^{2}+a^{2}}\left(\frac{\partial}{\partial r}\right)^{\mu}+\frac{\Delta a \sin ^{2} \theta}{2\left(r^{2}+a^{2}\right)}\left(\frac{\partial}{\partial \phi}\right)^{\mu} \\
& n_{\mu}=\frac{r^{2}+a^{2}}{\rho^{2}}\left(-\frac{\partial}{\partial v}+a \sin ^{2} \theta \frac{\partial}{\partial \phi}\right)
\end{aligned}
$$

where $\Delta=\left(r-r_{+}\right)\left(r-r_{-}\right)$and $r_{ \pm}=M \pm \sqrt{M^{2}-a^{2}}$ The null vectors satisfy the following conditions $l^{\mu} n_{\mu}=-1, l^{\mu} l_{\mu}=$ $n^{\mu} n_{\mu}=0$.

The covariant divergence of outgoing null vector field is given by

$$
\theta_{l}=\nabla \cdot \bar{l}=\frac{l^{r}}{\sqrt{g}} \frac{\partial}{\partial r} \sqrt{g}=\frac{\Delta r}{\rho^{2}\left(r^{2}+a^{2}\right)}
$$

The covariant divergence of ingoing null vector field is also given by

$$
\theta_{n}=\nabla \cdot \bar{n}=\frac{n^{r}}{\sqrt{g}} \frac{\partial}{\partial r} \sqrt{g}=-\frac{2 r\left(r^{2}+a^{2}\right)}{\rho^{4}}
$$

So the divergence of the ingoing null vector $\theta_{n}$ will be negative everywhere and the divergence of the outgoing null vector $\theta_{l}$ will change sign at the horizon $\Delta=0$. Thus the surface $\Delta=0$ will be a marginally trapped surface. In the region $r_{-}<r<r_{+}, \theta_{l}<0$ for $\Delta<0$. This implies that trapped surfaces exist for non extreme Kerr black hole in this region (see figure 1). In contrast, for the extreme Kerr black hole i.e. when $a=M$, we have the outgoing and ingoing covariant divergences to be

$$
\theta_{l}=\frac{r(r-M)^{2}}{\rho^{2}\left(r^{2}+a^{2}\right)}, \theta_{n}=-\frac{2\left(r^{2}+a^{2}\right)}{\rho^{4}}
$$

Here inside or outside extremal horizon that is $r<M$ or $r>M, \theta_{l}>0$ and $\theta_{n}<0$. This implies that there are no trapped surfaces for the extreme Kerr black hole beyond the event horizon. For $r=M$, the extremal horizon is a marginally trapped surface.

\subsection{Trapped Surface in Kerr Black Hole Using the Flux of a Vector Field}

The flux of outgoing null vector field $l$ is given by

$$
\phi_{l}=\frac{2 \pi G M \triangle}{\left(r^{2}+a^{2}\right)}
$$

The flux of ingoing null vector field $n$ is given by

$$
\phi_{n}=-\frac{4 \pi G M\left(r^{2}+a^{2}\right)}{\rho^{2}}
$$

From these equations, the flux of the ingoing null vector $\phi_{n}$ is negative everywhere and that of outgoing null vector $\phi_{l}$ will change sign at the horizon $\Delta=0$. Thus the surface $\Delta=0$ will be a marginally trapped surface. For $\Delta<0, \theta_{l}<0$ and $\theta_{n}<0$. This implies trapped surfaces exist in the region $r_{-}<r<r_{+}$for the Kerr black hole. In contrast, for the extreme Kerr black hole i.e. when $a=M$, the fluxes of outgoing null vector and ingoing null vector are respectively given by

$$
\begin{aligned}
& \phi_{l}=\frac{2 \pi G M(r-M)^{2}}{\left(r^{2}+a^{2}\right)} \\
& \phi_{n}=-\frac{4 \pi G M\left(r^{2}+a^{2}\right)}{\rho^{2}}
\end{aligned}
$$


Here inside or outside extremal horizon that is $r<M$ or $r>M, \phi_{l}>0$ and $\phi_{n}<0$. But, for $r=M, \phi_{l}=0$ implying that the extremal horizon is a marginally trapped surface. This implies that there are no trapped surfaces for extremal Kerr black hole beyond the event horizon. This shows that the covariant divergence and the flux being related due to the divergence theorem it follows that the flux can also be used to define trapped and marginally trapped surfaces.

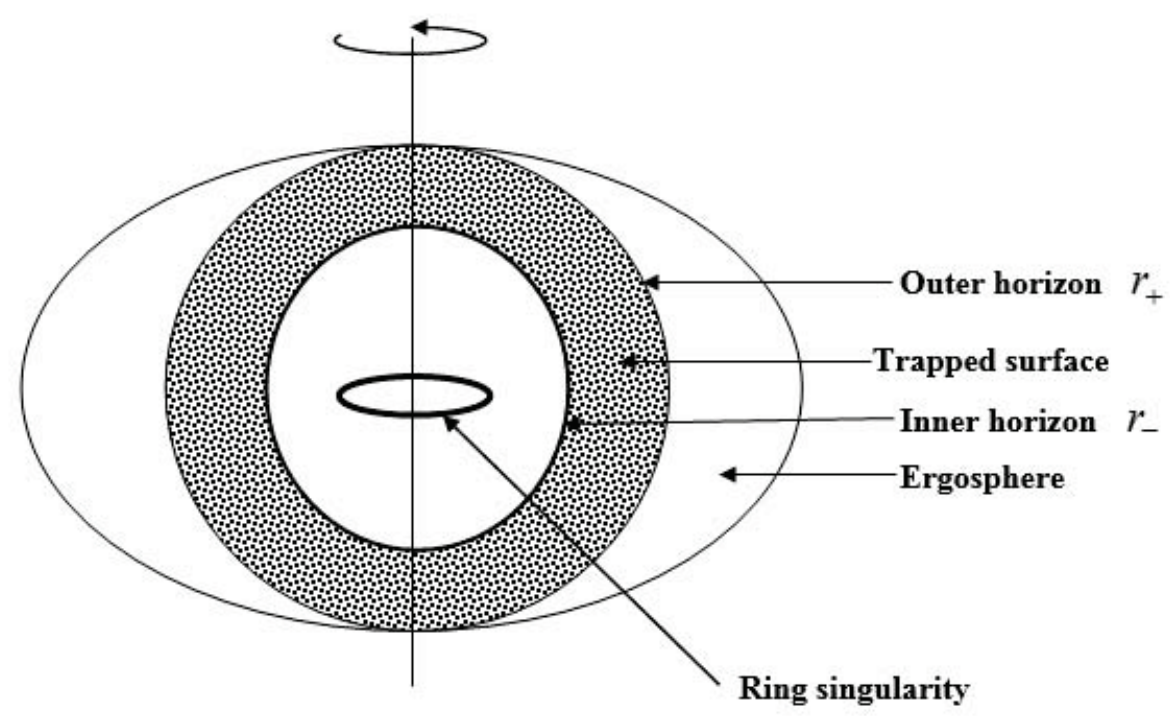

Figure 1. The locations of the event horizons, the ring singularity, ergosphere and trapped surface which lies in the region $r_{-}<r<r_{+}$in Kerr the black hole

\section{Conclusion}

Trapped and marginally trapped surfaces play a very important role in the analysis of spacetime geometry. By the Hawking and Penrose singularity theorems, a spacetime which satisfies suitable energy and causality conditions and which also contains a trapped surface, must contain a black hole (Hawking and Ellis, 1973). We have by this paper explicitly demonstrated this theorem. The computations of the divergences and the fluxes of the outgoing and ingoing null vectors have revealed that the Kerr black hole region contains trapped surfaces, a closed two-surfaces $\mathrm{S}$ with the property that for both ingoing and outgoing null vectors orthogonal to $S$, the divergences and the fluxes are negative everywhere on $S$. An exception is extreme Kerr black hole which does not contain any trapped surface. The extremal horizon is marginally trapped surface. Since these computations have confirmed the existence of trapped and marginally trapped surfaces in the Kerr spacetime, it is therefore desirable to use trapped surfaces as a suitable complement in studying black holes. An advantage of, using trapped surfaces as local characterizations of black holes is that they do not depend on global properties like the classical event horizon whose determination requires the knowledge of the entire future null infinity.

\section{Acknowledgment}

We thank God for His guidance throughout this work and we are also grateful to the late Prof. FKA Allotey (1932 - 2017) for his encouragement and mentorship 


\section{References}

Ashtekar, A., \& Krishnan, B. (2004). Living Rev. Rel., 7, 10. (www.livingreviews.org/lrr-2004-10).

Ashtekar, A., \& Galloway Gregory, J. (2005). Some uniqueness results for dynamical horizons. International Press Adv. Theor. Math. Phys., 91C30.

Ashtekar, \& Krishnan, B. (2004). Isolated and Dynamical Horizons and Their Applications, Living Rev. Relativity 710 URL.

Ben-Dov, I. (2007). ”outer trapped surfaces in Vaidya spacetimes” arxiv:grqc/ 0611057.

Booth, I. (2005). Black hole boundaries, Can. J. phys. 831073.

Booth, I., Kunduri, H. K., \& O’Grady, A. (2017). Unstable marginally outer trapped surfaces in static spherically symmetric spacetimes: Physics Reviewed D, 96.

Borisenko, A. I., \& Tarapov, I. E. (1979). Vector and Tensor analysis with applications, Dover publications Inc. New York.

Carroll, S. (2014). Spacetime and Geometry, an introduction to General Relativity. Pearson Education Limited, Edinburgh Gate, Harlow, Essex CM202JE

Chandrasekhar, S. (1983). The mathematical theory of black holes; Oxford University press. New York

Frolov Valeri, P., \& Andrei, Z. (2011). Introduction to Black hole physics; Oxford University press, New York.

Gourgoulhon, E., \& Jose Luis, J. (2008). New theoretical approaches to black holes, arXiv.org.

Gourgoulhon, E. (2017). Geometry and physics of black holes, Lecture notes.

Hartle James, B. (2003). An introduction to Einsteins General Relativity, Addison Wesley, San Francisco.

Hawking, S. W., \& Ellis, G. F. R. (1973). The Large Scale Structure of Spacetime, Cambridge University Press, Cambridge.

Hawking, S. W., \& Penrose, R. (1970). The Singularities of Gravitational Collapse and Cosmology, Proc. R. Soc. Lond. A vol. 314 no. 1519 529-548. https://doi.org/10.1098/rspa.1970.0021

Hayward, S. A. (1994). “General laws of black hole dynamics” phys. Rev. D49 6467 [arXiv; gr-qc/9303006].

Jakobsson, E. (2017). Shapes of Spacetimes - Collected tales of black holes, Department of Physics, Stocholm University.

Jaramillo Jos, L. (2011). An introduction to local black hole horizons in the 3+1 approach to general relativity, MaxPlanck-Institute f ur Gravitations physik, Albert Einstein Institut, Am Mhlenberg 1, Golm D-14476, Germany.

Krishnan, B. (2012). The spacetime in the neighborhood of a general isolated black hole. Class. Quant. Grav. 29:205006.

Krishnan, B. (2013). Quasi-local black hole horizons, Max Planck Institute for Gravitational Physics (Albert Einstein Institute), Callinstr. 38, 30167 Hannover, Germany.

Kumah, M., \& Oduro, F. T. (2018). On the trapped surface characterization of black hole region in Vaidya spacetime. Journal of Mathematics Research, Canadian Center of Science and Education; 10(1), 1-8.

O’Neill, B. (1995). The geometry of Kerr black holes, A K Peters, Ltd 289 Linden Street Wellesley, M A 02181.

Penrose, R. (2004). The road to reality. A complete guide to the laws of the universe; Published by Jonathan Cape.

Penrose, R. (1965). Gravitational collapse and space-time singularities, Phys. Rev. Lett. 1457.

Schnetter, E., \& Krishnan, B. (2006) "Non-symmetric trapped surfaces in the Schwarzschild and Vaidya spacetimes" Phys. Rev. D73 021502 [arXiv: gr-qc/0511017].

Senovilla Jose, M. M. (2011). Trapped surfaces; arXiv-1107.1344, International Journal of Modern Physics: Conference Series World Scientific Publishing Company.

Senovilla Jos, M. M. (1998a). "Singularity Theorems and Their Consequences” (Review) General Relativity and Gravitation $30701-848$.

Senovilla Jose, M. M., \& Garnkle, D. (2015). The 1965 penrose singularity theorem. Classical and Quantum Gravity, 32. https://doi.org/10.1088/0264-9381/32/12/124008 


\section{Copyrights}

Copyright for this article is retained by the author(s), with first publication rights granted to the journal.

This is an open-access article distributed under the terms and conditions of the Creative Commons Attribution license (http://creativecommons.org/licenses/by/4.0/). 